\title{
A unique "fishtail-like” four-way shape memory effect of compositionally graded NiTi
}

Qinglin Meng ${ }^{1}$, Zhigang $\mathrm{Wu}^{1 *}$, Reza Bakhtiari ${ }^{1}$, Bashir S. Shariat ${ }^{1}$, Hong Yang ${ }^{1}$, Yinong Liu ${ }^{1 *}$, and Tae-hyun $\mathrm{Nam}^{2}$

\author{
1 School of Mechanical and Chemical Engineering, The University of Western Australia, \\ Crawley, WA 6009, Australia \\ 2 School of Materials Science and Engineering \& RIGET, Gyeongsang National University, \\ 900 Gazwadong, Jinju, Gyeongnam 660-701, Republic of Korea
}

\begin{abstract}
This paper reports a complex "four-way" shape memory effect of a compositionally graded NiTi thin plate. The composition gradient is created by surface diffusion of Ni into the plate. After a $15 \%$ tensile deformation pre-treatment, the compositionally graded plate exhibits a complex and novel four-way shape memory effect in bending mode, by which the metal exhibits a back-and-forth shape change upon a single heating or cooling, thus displaying four shape changes over one complete thermal cycle. Such behavior, which is analogous to a "fishtail-like" motion, results from the sequential transformation through the thickness of the thin plate as dictated by the Ni content gradient of the alloy. Such "four-way" shape memory effect has not been achieved in NiTi alloys before.
\end{abstract}

Keywords: Shape memory alloy (SMA); Functionally graded materials (FGM); Two-way shape memory effect

This paper reports on a new "four-way" shape memory effect, by which the metal exhibits a back-and-forth shape change upon a single heating or cooling, thus displaying four shape changes over one complete thermal cycle. Such behavior, which is analogous to a fishtail-like motion, is achieved in a functionally graded NiTi plate. In this behaviour, the thin NiTi plate bends forth and then back subsequently in two directions upon one cooling or heating, thus exhibits a unique "four-way" memory effect over a full cooling-heating cycle without the assistance of any external stress. Such behaviour, which has not been reported in the literature, enriches the functionality of shape memory alloys.

Automatic reversible shape change, conventionally known as "two-way shape memory effect" (TWSME), originates from the phase transformation between the low temperature martensite phase and high temperature austenite phase [1, 2]. It is an essential property for self-actuated shape memory devices, where cyclic actuation under temperature-controlled mechanism is involved, e.g., the microwrapper [3] and biomimic robotic hands [4]. A TWSME component is more advantageous in design than using a bias spring [5, 6] for shape re-setting after each action, by being simple and more adaptable to miniature designs and complex motions.

The TWSME of NiTi alloy is usually developed through certain thermomechanical treatments, known as training. Typical training schemes are repeated cycles in pseudoelastic deformation [7] thermal transformation under constant stress [8-10] or combinations of 
temperature variation and deformation [11]. It is commonly accepted that the TWSME originates from the anisotropic dislocation structures developed in the austenite matrix during the prior training process. The anisotropic internal stress fields associated with such oriented dislocations induce the formation of preferentially oriented martensite variants during subsequent thermal transformation cycles [7-13]. Simple deformation via martensite reorientation or stress-induced martensite transformation of NiTi to well beyond the stress plateau has also proven to be effective in developing TWSME [14-17]. For Ni-rich alloys, constrained aging is also able to develop TWSME, commonly known as "all-round" shape memory effect [18-21]. In this work we developed a new form of "all-round" shape memory effect featuring a novel "four-way" memory cycle using a functionally graded NiTi thin plate.

A commercial Ti-50.07 at.\% Ni thin plate strips of $40 \times 5 \times 0.22 \mathrm{~mm}$ in dimension was used in this study. The strips were annealed at $1123 \mathrm{~K}$ for $3.6 \mathrm{ks}$ in vacuum. A Ni thin film of $\sim 500$ $\mathrm{nm}$ in thickness was deposited by means of DC magnetron sputtering under argon protection. The coated NiTi strips were then annealed at $1223 \mathrm{~K}$ in vacuum for $10.8 \mathrm{ks}$ to encourage diffusion of the Ni into the NiTi plate [22]. The inset in Figure 1(a) shows schematic of the Ni content distribution though the thickness of the plate samples before and after diffusion annealing. The actual distribution of $\mathrm{Ni}$ content through the thickness after the diffusion anneal was determined by x-ray energy dispersive spectrometry (EDS) analysis, which is shown in Figure 1(b). It is seen that the anneal created a compositional gradient from 50.5 to 50.00 at.\% Ni through the thickness of the plate.
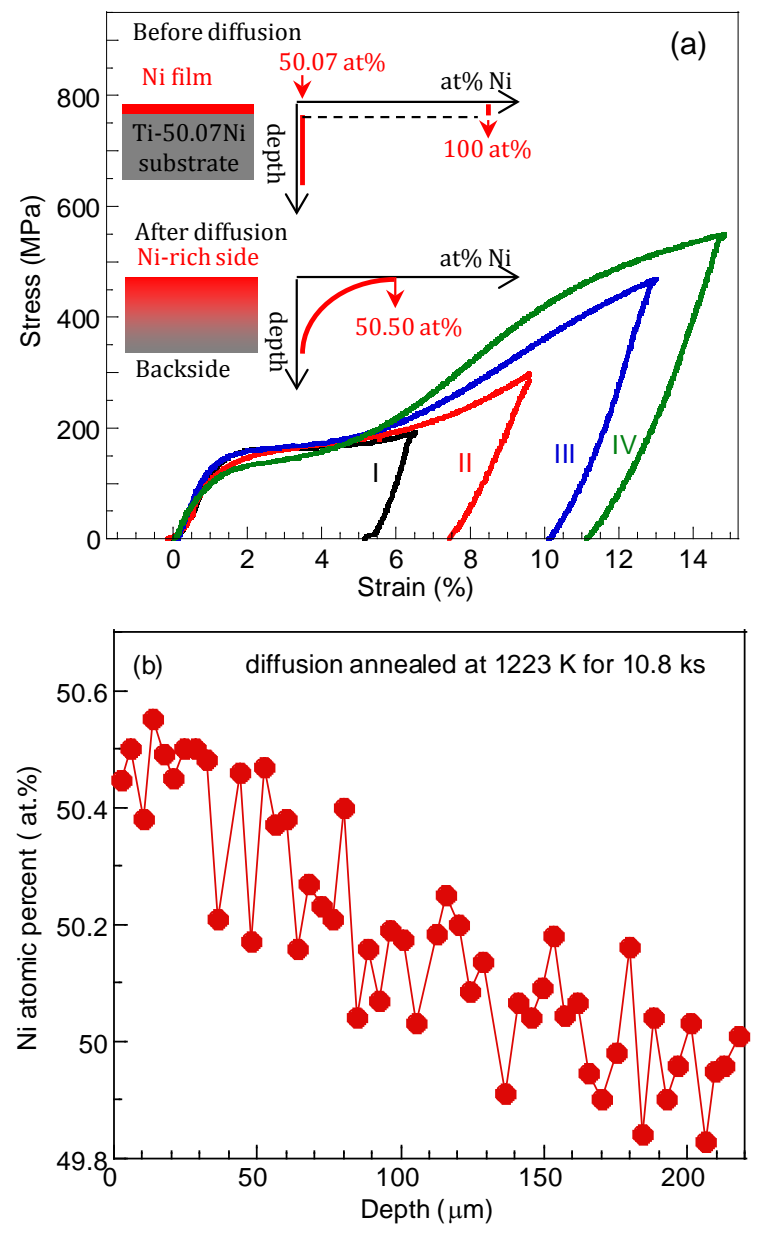

Figure 1. Compositionally graded NiTi thin plate; (a) Tensile deformation behaviour; (b) 
Diffusion induced Ni content distribution through the thickness as determined by EDS analysis.

The transformation behaviour of the plate was analysed by differential scanning calorimetry (DSC), and the parameters were determined to be $M_{s}=302 \mathrm{~K}, M_{f}=231 \mathrm{~K}$ and $\Delta H^{A-M}=$ $22.2 \mathrm{~J} / \mathrm{g}$ for the forward and $A_{s}=251 \mathrm{~K}, A_{f}=328 \mathrm{~K}$ and $\Delta H^{M-A}=22.6 \mathrm{~J} / \mathrm{g}$ for the reverse transformation. The very wide transformation temperature intervals $\left(M_{f}-M_{s}=71 \mathrm{~K}\right.$ and $A_{f}$ $A_{s}=77 \mathrm{~K}$ ) are unusual and are a manifestation of the composition gradient through the thickness of the plate. It is apparent that the $M_{s}(302 \mathrm{~K})$ corresponds to the start temperature of the martensitic transformation at the back side (where the alloy is of the original composition of 50.07 at.\% $\mathrm{Ni})$ and the $M_{f}(231 \mathrm{~K})$ corresponds to the finishing temperature of the martensitic transformation at the coated side (the Ni-rich side where the alloy composition is 50.47 at.\% $\mathrm{Ni}$ ).

The strip samples were deformed in tension to various strains using an Instron 4301 universal testing machine at a strain rate of $8.33 \times 10^{-5} \mathrm{~s}^{-1}$ at room temperature $(300 \mathrm{~K})$, with a $20 \mathrm{~mm}$ gauge length. The samples were cooled in liquid nitrogen prior to deformation to maximise the volume fraction of martensite structure at the tensile test temperature (room temperature). Prior to tensile testing, the strip samples contained both austenite and martensite with the austenite portion existing on the Ni-rich side and the martensite portion on the back side. Figure 1(a) shows the tensile stress-strain curves of four samples deformed to $6.5 \%, 10 \%$, $12.5 \%$ and $15 \%$ tensile strains, labelled as sample I, II, III and IV, respectively. The deformation over the transformation plateau occurred in a mixed mode of martensite variant reorientation in the region close to the back side and stress-induced transformation in the region close to the Ni-rich side, at $\sim 160 \mathrm{MPa}$ of stress and to $6 \%$ of strain. After unloading, all four samples showed a slight bend towards the Ni-rich side. This is because that the Nirich side was in austenitic state with a martensitic transformation temperature well below the room temperature, thus upon unloading this part experienced some pseudoelastic recovery through the reverse martensitic transformation. Consequently, the Ni-rich side surface contracted for a small amount while the back side remained the same length following unloading, thus forming a curvature towards the Ni-rich side.

After deformation, the NiTi strips were subjected to heating and cooling cycles in hot water and chilled ethanol baths and the shape changes of the strip samples were recorded using a digital camera. The curvature of the bent shapes of the strips was determined from the recorded images using Image $\mathrm{J}$ (NIH freeware), defined as $\kappa=\frac{1}{R}$, where $\mathrm{R}$ is the radius of the curve. The curvature is denoted positive when the plate bends towards the Ni-rich side and negative if it bends towards the back side. The surface strain was computed as $\varepsilon_{s}=\frac{d_{N i T i}}{R}$, where $d_{N i T i}$ is the plate thickness.

Figure 2(a) shows the effect of heating temperature on the evolution of the curvature and surface strain of the four deformed samples. Upon the first heating, all four samples showed "bell-shaped" $\kappa-\mathrm{T}$ curves, i.e., increasing and then decreasing in curvature with increasing temperature. The increase of the curvature is due to the occurrence of the reverse 
transformation (contraction) first on the Ni-rich side, where the transformation temperature is the lowest [23]. With the propagation of the reverse transformation through the thickness from the Ni-rich side to the back side upon further heating, the region near the back side started to transform which also resulted in the shortening of sample length, causing reverse bending (curving) of the strip sample. Due to the greater amount of contraction on the back side comparing to the Ni-rich side, the curvature flipped to the opposite side eventually.

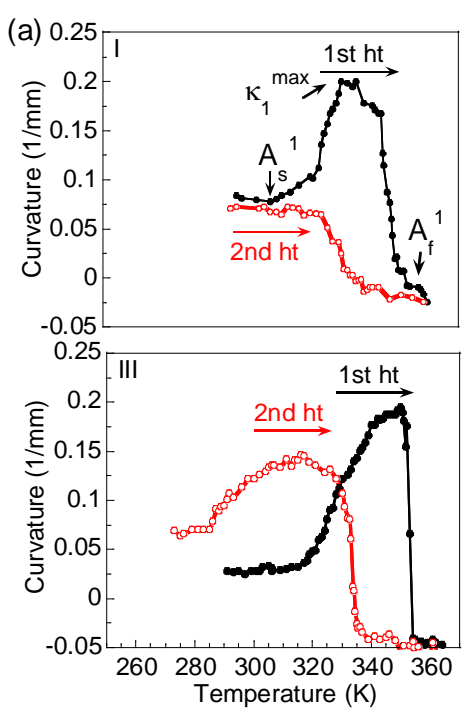

(b) $\quad$ increase
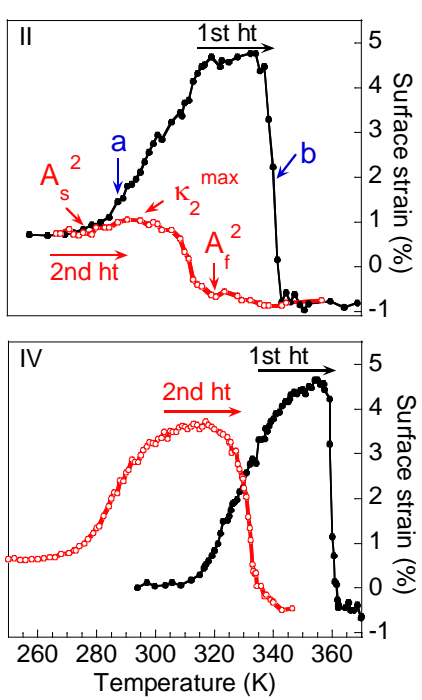

Curvature decrease

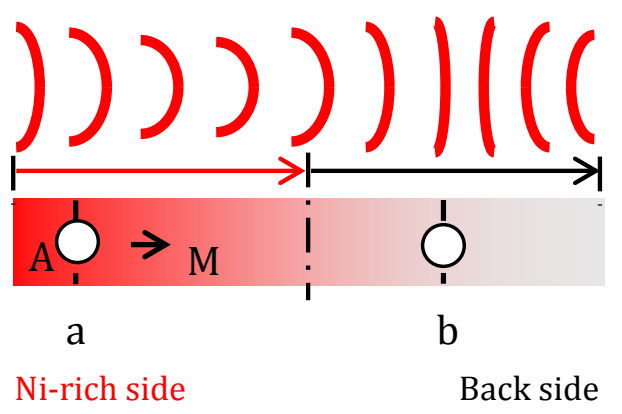

Figure 2. (a) Effect of temperature on the evolutions of curvature and surface strain upon the 1st and 2nd heating of the four deformed NiTi plates; (b) Schematic of the curvature change as a result of the propagation of the reverse martensitic transformation from the Ni-side to the back side upon heating; $\mathrm{A}$ and $\mathrm{M}$ denote austenite and martensite, respectively.

Figure 2(b) shows a schematic of the propagation of the reverse transformation front through the thickness and its effect on sample curvature. Marks "a" and "b" indicate the positions of the reverse transformation front at two moments during the process of the reverse transformation which are also marked in Figure 2(a)-II. At moment "a", the left part (the Nirich side) has transformed back to austenite, and the sample curves up to the Ni-rich side. At moment (b), the transformation has propagated through to the back side, causing the sample to curve back, eventually into a slight negative curvature at the completion of the transformation.

Shape change upon the second heating is the two-way memory effect. It is seen that upon the second heating, samples I and II showed a monotonic curvature decrease. This is indicative that the samples strips have developed two-way memory effect only on the back side, thus the 
samples bent only in the negative direction upon cooling and heating. These two samples had been deformed to lower levels of pre-deformation, which is apparently insufficient to induce effective two-way effect in the Ni-rich side of the strips. In comparison, samples III and IV showed the reversible "bell-shaped" recovery. This demonstrates that the samples have developed two-way memory effect throughout the thickness, in both the back side and the Nirich side. The range of the curvature changes of the second heating (between the maximum and the minimum curvatures) of the four samples are measured to be $\Delta \kappa_{I}=0.07(1 / \mathrm{mm})$, $\Delta \kappa_{I I}=0.09(1 / \mathrm{mm}), \Delta \kappa_{I I I}=0.18(1 / \mathrm{mm})$, and $\Delta \kappa_{I V}=0.19(1 / \mathrm{mm})$ for the four samples, and the maximum two-way memory (surface) strain range achieved was $4.15 \%$ as in sample IV. It is also evident that the transformation temperatures on the first heating ( $A_{s}^{1}$ and $A_{f}^{1}$ ) are higher than those on the second heating $\left(A_{s}^{2}\right.$ and $A_{f}^{2}$ ). This is due to the deformation induced martensite stabilisation effect [14, 17, 24, 25].

The effect of pre-strain on the maximum curvature achieved on the first heating $\left(\kappa_{1}^{\max }\right)$ and the maximum curvature achieved on the second heating $\left(\kappa_{2}^{\max }\right)$. The ratio $\eta^{t w}=\frac{\kappa_{2}^{\max }}{\kappa_{1}^{\max }} \times 100 \%$ defines the efficiency of the two-way memory effect. It is seen from Figure 2(a) that $\kappa_{1}^{\max }$ remained practically constant at $\sim 0.20(1 / \mathrm{mm})$ independent of pre-strain (at>6.5\%). In contrast, $\kappa_{2}^{\max }$ increased continuously with increasing pre-strain, reaching a maximum $\eta^{t w}=$ $81 \%$ at $15 \%$ pre-strain. This value is comparable to results achieved by conventional training [12-15].

Figure 3 shows collections of optical images of samples IV during the 20th heating/cooling thermal cycle after the tensile deformation to $15 \%$. Each illustration is a montage of separate optical images of the same plate sample upon heating to different temperatures, constructed using Photoshop (C). The images show the edge of the thin plate. For all the images, the Ni rich side is to the right, as indicated by the arrow. Illustrations C(1) and C(2) are for cooling from $315 \mathrm{~K}$ to $283 \mathrm{~K}$ and from $276 \mathrm{~K}$ to $223 \mathrm{~K}$, respectively. It is seen that the sample started with a slight negative curvature, bending towards the Ni-rich side upon cooling to a maximum curvature and then backwards to a slightly positive curvature with further cooling to the end. Similarly, illustrations $\mathrm{H}(1)$ and $\mathrm{H}(2)$ are for heating from $251 \mathrm{~K}$ to $308 \mathrm{~K}$ and from $308 \mathrm{~K}$ to $345 \mathrm{~K}$, respectively. 


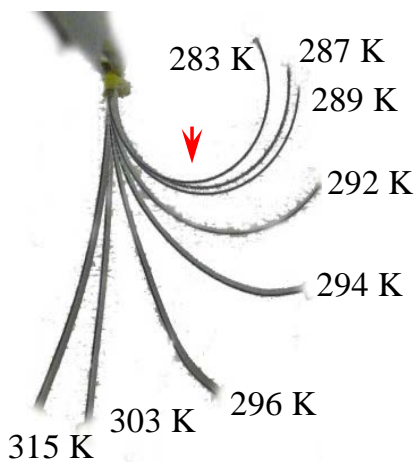

C(1)
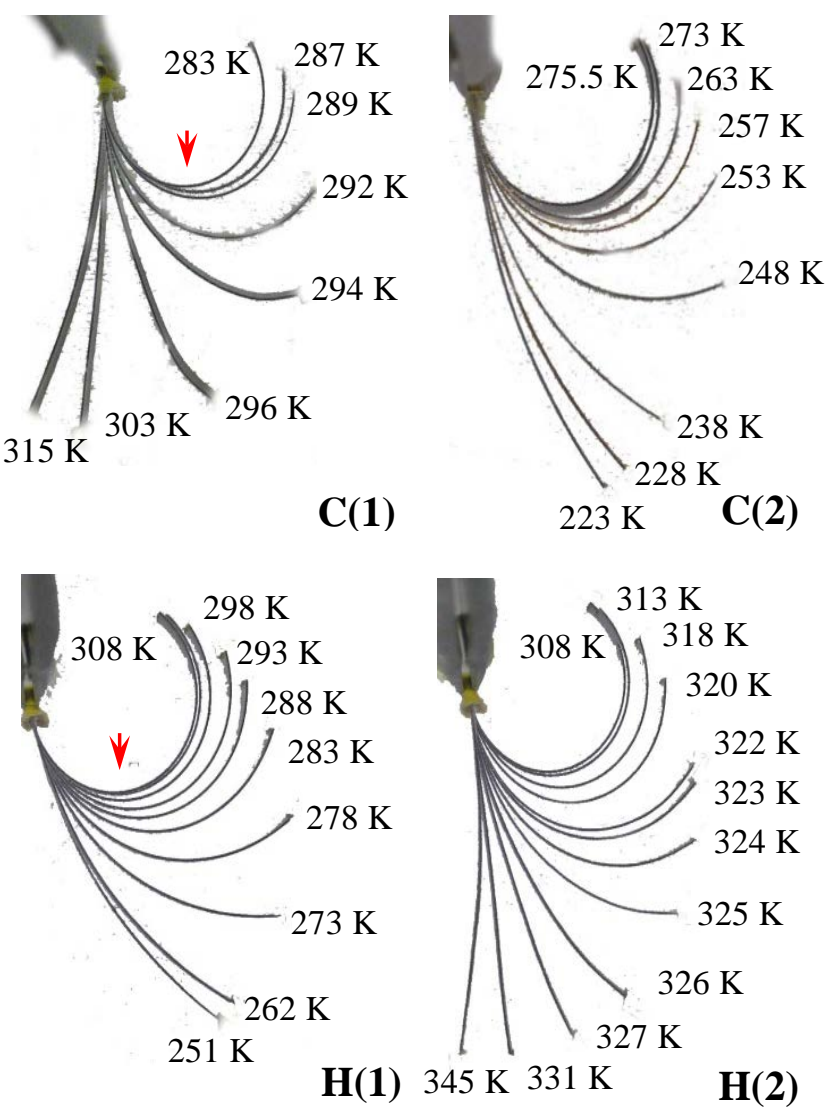

Figure 3. Optical image montages showing shape evolution of compositionally graded NiTi thin plates with $15 \%$ pre-strain in tension.

Figure 4 shows the complete two-way memory cycles of samples III and IV during the 20th thermal cycle, expressed as the evolution of curvature and surface strain on temperature. Sample III showed a hysteresis shape change curve similar to that of conventional NiTi, but with a slight reversible shape change (increase and then decrease or vice versa) upon one heating or cooling. In comparison, sample IV showed a complete shape change cycle with two shapes achieved on each heating or cooling, with the double "bell-shaped" curves. The range of curvature change of the two samples are determined to be $\Delta \kappa_{I I I}=0.16(1 / \mathrm{mm})$ for sample III and $\Delta \kappa_{I V}=0.18(1 / \mathrm{mm})$ for sample IV. These examples (and the results presented in Figure 1) demonstrate that complex two-way memory effect in bending can be easily introduced by simple tensile deformation, thanks to the composition gradient in the plate, and that the extent of the bending can be easily tailored by controlling the level of pre-strain of the tensile deformation. 

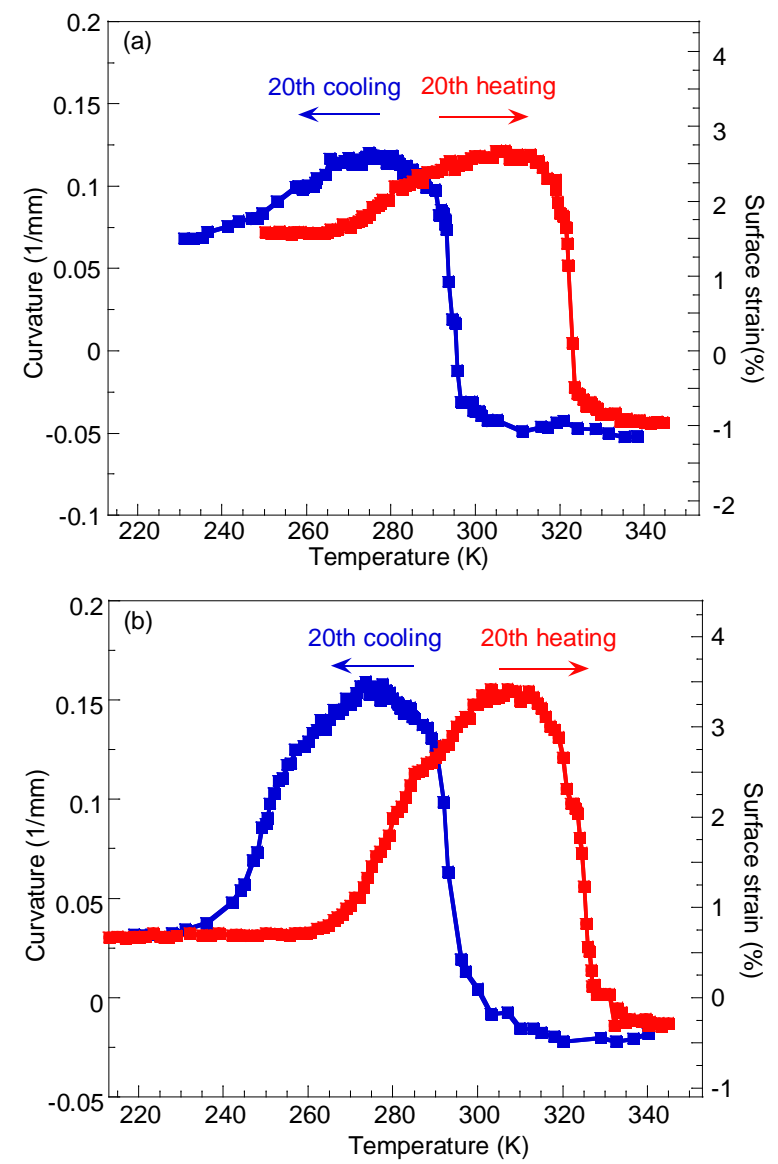

Figure 4. Evolution of curvature and surface strain upon the $20^{\text {th }}$ thermal cycle for compositionally graded NiTi plate subjected to (a) $12.5 \%$ and (b) $15 \%$ of pre-deformation, demonstrating the complex two-way shape memory effect.

The complex bending-unbending shape evolution of the compositionally graded NiTi thin plate resembles a "fishtail-like" motion. Such behaviour is unique. No similar observation has been reported in the literature. The only similar shape memory motion reported is from a complex design of multilayer phase-coupled SMA thin film composite involving TiNiCu, TiNiHf and Mo [5]. Other observations of two-way memory effect in bending mode all involve monotonic shape changes in each heating or cooling direction. This is achieved by constrained aging (constrained in bending) of Ni-rich NiTi thin plates [21, 26, 27], which induces formation of lenticular $\mathrm{Ti}_{3} \mathrm{Ni}_{4}$ precipitates in perpendicular orientations on the compressed and elongated sides of the bent plate. Similar results are also achieved for NiTi thin films by the same technique of constrained ageing $[18,20]$. Lehnert et al. reported an interesting work on $\mathrm{Ni} / \mathrm{Ti}$ multilayer thin films, which are converted into NiTi alloy films by diffusion anneal [19]. In this work they proposed the concept of creating composition gradient in thickness of the film by controlling the relative thicknesses of the $\mathrm{Ni}$ and $\mathrm{Ti}$ layers during film deposition. In this work they reported the spontaneous (monotonic) two-way memory effect as a rare observation among their multilayer NiTi thin films, and attributed the effect to intrinsic internal stresses. Considering all these, it is seen that such a complex, "4way" spontaneous shape memory effect in a "fishtail-like" motion in bending has not been achieved so far in monolithic NiTi materials. Coupled with the wide temperature window for the shape change, typically $\sim 70 \mathrm{~K}$, it offers new opportunities for innovative designs with 
ease of control to reach desired positions using these specially developed functionally graded NiTi alloys.

In conclusion, a complex "4-way" shape memory effect is achieved in compositionally graded NiTi thin plates. The effect features reversible "fishtail-like" motion in stress-free thermal cycles and wide actuation temperature windows. The effect is induced by a simple tensile deformation. The parameters of the complex shape memory effect can be tailored by controlling the level of pre-strain in the tensile deformation.

This work was supported by the Australian Research Council in grants ARC-DP140103805 and ARC-DE150101795.

[1] R. Rapacioli, V. Torra, E. Cesari, Scr. Metall. 22 (1988) 261-264.

[2] C. M. Wayman, I. Cornelis, Scr. Metall. 6 (1972) 115-122.

[3] Y. Fu, H. Du, W. Huang, S. Zhang, M. Hu, Sens. Actuator A 112 (2004) 395-408.

[4] D. Reynaerts, H. V. Brussel, Mechatron. 8 (1998) 635-656.

[5] B. Winzek, S. Schmitz, H. Rumpf. T. Sterzl, R. Hassdrof, S. Thienhaus, J. Feydt, M. Moske, E. Quandt, Mater. Sci. Eng. A 378 (2004) 40-46.

[6] K. Kuribayashi, T. Taniguchi, M. Yositake, S. Ogawa, Mat. Res. Soc. Symp. Pro. 276 (1992) 167.

[7] L. Contardo, G. Guenin, Acta Metall. Mater. 38 (1990) 1267-1272.

[8] R. Lahoz, J. A. Puértolas, J. Alloy Compd. 381 (2004) 130-136.

[9] Z. Bo, D. C. Lagoudas, Inter. J. Eng. Sci. 37 (1999) 1175-1203.

[10] K. Wada, Y. Liu, J. Alloy Compd. 449 (2008) 125-128.

[11] J. Perkins, R. O. Sponholz, Metall. Trans. A15 (1984) 313-321.

[12] Y. Liu, P. G. McCormick, Acta Metall. Mater. 38 (1990) 1321-1326.

[13] R. Stalmans, J. V. Humbeeck, L. Delaey, Acta Metall. Mater. 40 (1992) 501-511.

[14] Y. Liu, Y. Liu, J. V. Humbeeck, Acta Mater. 47 (1999) 199-209.

[15] Z. Xie, Y. Liu, J. V. Humbeeck, Acta Mater. 46 (1998) 1989-2000.

[16] Y, Liu, D. Favier, Acta Mater. 48(2000) 3489-3499.

[17] G. Tan, Y. Liu, Intermet. 12 (2004) 373-381.

[18] A. Gyobu, Y. Kawamura, H. Horikawa, T. Saburi, Mater. Sci. Eng. A 273-275 (1999) 749-753.

[19] T. Lehnert, H. Grimmer, P. Böni, M. Horisberger, R. Gotthardt, Acta Mater. 48 (2000) 4065-4071.

[20] A. Gyobu, Y. Kawamura, T. Saburi, M. Asai, Mater. Sci. Eng. A 312 (2001) 227-231.

[21] M. Nishida, T. Honma, Scr. Metall. 18 (1984) 1299-1302.

[22] Q. Meng, H. Yang, Y. Liu, T. Nam, Scr. Mater. 67 (2012) 305-308.

[23] J. Frenzel, E. P. George, A. Dlouhy, Ch. Somsen, M. F. Wagner, G. Eggeler, Acta Mater. 58 (2010) 3444-3458.

[24] C. Picornell, J. Pons, E. Cesari, Acta Mater. 49 (2001) 4221-4230.

[25] M. Piao, K. Otsuka, S. Miyazaki, H. Horikawa, Mater. Trans. JIM 34 (1993) 919-929.

[26] R. Kainuma, M. Matsumoto, Scr. Matall. 22 (1988) 475-478.

[27] M. Nishda, T. Honma, Scr. Metall. 18 (1984) 1293-1298. 